\title{
DC Characteristics Optimization of a Double G-Shield 50 V RF LDMOS
}

\author{
Xiangming Xu, ${ }^{1,2}$ Pengliang Ci, ${ }^{2}$ Xiaoyu Tang, ${ }^{2,3}$ Jing Shi, ${ }^{2}$ \\ Zhengliang Zhou, ${ }^{2}$ Jingfeng Huang, ${ }^{2}$ Peng-Fei Wang, ${ }^{1}$ and David Wei Zhang ${ }^{1}$ \\ ${ }^{1}$ State Key Laboratory of ASIC and System, School of Microelectronics, Fudan University, Shanghai 201203, China \\ ${ }^{2}$ Huahong Grace Semiconductor Manufacturing Corporation, Shanghai 201203, China \\ ${ }^{3}$ School of Electronic Science and Engineering, Nanjing University, Nanjing 210093, China
}

Correspondence should be addressed to Peng-Fei Wang; pfw@fudan.edu.cn

Received 21 October 2014; Accepted 4 November 2014

Academic Editor: Rui Zhang

Copyright (C) 2015 Xiangming Xu et al. This is an open access article distributed under the Creative Commons Attribution License, which permits unrestricted use, distribution, and reproduction in any medium, provided the original work is properly cited.

An N-type 50 V RF LDMOS with a RESURF (reduced surface field) structure of dual field plates (grounded shield, or G-shield) was investigated. The effect of the two field plates and $\mathrm{N}$-drift region, including the junction depth and dopant concentration, on the DC characteristics was analyzed by employing the Taurus TCAD device simulator. A high BV (breakdown voltage) can be achieved while keeping a low $R_{\mathrm{DSON}}$ (on-resistance). The simulation results show that the $\mathrm{N}$-drift region dopant concentration has an obvious effect on the BV and $R_{\mathrm{DSON}}$ and the junction depth affected these values less. There is an optimized length for the second field plate for a given dopant concentration of the $\mathrm{N}$-drift region. Both factors should be optimized together to determine the best $\mathrm{DC}$ characteristics. Meanwhile, the effect of the first field plate on the $\mathrm{BV}$ and $R_{\mathrm{DSON}}$ can be ignored. According to the simulation results, $50 \mathrm{~V}$ RF LDMOS with an optimized RESURF structure of a double G-shield was fabricated using $0.35 \mu \mathrm{m}$ technologies. The measurement data show the same trend as the TCAD simulation, where a BV of $118 \mathrm{~V}$ and $R_{\mathrm{DSON}}$ of $26 \mathrm{ohm} \cdot \mathrm{mm}$ were achieved.

\section{Introduction}

A RF LDMOS (radio frequency lateral double diffused metal oxide silicon) device is a competitive power device. It was initially used to displace the bipolar transistor for cellular infrastructure applications $[1,2]$. It achieves good linearity, high gain, high breakdown voltage, high output power, high efficiency, good thermal stability, and good broadband match characteristics and is easy to integrate with the MOS process $[3,4]$. It is also much cheaper than GaAs devices. It can cover a frequency range of $1 \mathrm{MHz}$ to $4 \mathrm{GHz}[5,6]$. Because of these merits, it is widely used as a power amplifier in GSM, PCS, and W-CDMA cellular infrastructure, wireless broadcast, industry, science, medical ISM, and radar $[7,8]$. Among them, a $50 \mathrm{~V}$ RFLDMOS device is mainly used in wireless broadcast, ISM, and radar, which require a higher breakdown voltage and power density $[9,10]$.

For a $50 \mathrm{~V}$ operation voltage device, the breakdown voltage must be higher than $110 \mathrm{~V}$ to guarantee reliable operation.
To obtain a high breakdown voltage and maintain a low onresistance, it is compulsory to adopt the RESURF structure. In RESULF technology, the vertical PN junction depletion layer between the diffusion layer and substrate and its interaction with the lateral PN junction depletion region between the channel and diffusion layer are used to reduce the peak value of the surface electrical field to obtain a higher breakdown voltage. For a $50 \mathrm{~V}$ device with a fixed diffusion length, to obtain a high breakdown voltage and low on-resistance, it is not sufficient to only adjust the electrical field distribution of the vertical and lateral PN junction. Introducing a field plate on top of the gate can reduce the feedback capacitance of the $C_{\mathrm{GD}}$ and increase the breakdown voltage of the device. The lateral and vertical PN junction distribution of the $\mathrm{N}$ type diffusion region is primarily determined by the $\mathrm{N}$ type implant energy and dose. The field-plate profile and the $\mathrm{N}$-type diffusion region underneath further adjust the electrical field distribution. The impact of the breakdown voltage and on-resistance from the $\mathrm{N}$-type diffusion region 


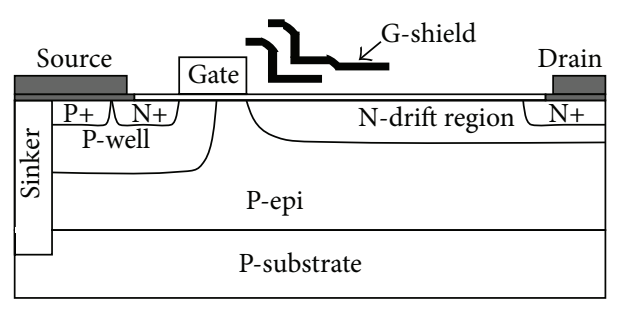

Figure 1: Diagram of the 50 V RFLDMOS device.

implant energy and dose and the length of dual field plate are investigated here. The best RESURF effect is achieved and confirmed by experiment. The device is optimized.

\section{Device Structure Analysis}

The breakdown voltage of $50 \mathrm{~V}$ operation voltage RLDMOS is determined both laterally and vertically. The vertical breakdown voltage is determined by the diffusion region depth and dopant concentration with the epitaxy layer thickness and dopant concentration. Apart from these factors, the lateral breakdown voltage is directly related to the N-type drift region length and surface electrical field distribution of the drift region. The thickness of a P-type epitaxy layer under an N-type drift region is normally sufficiently high that the device breakdown voltage is determined by the lateral breakdown. Figure 1 shows a structural illustration of the $50 \mathrm{~V}$ RFLDMOS device. Under the N-drift region is the Pepitaxy layer, which has a lower dopant concentration. Under a high drain voltage, the $\mathrm{N}$-drift region is fully depleted until the edge of the drain under a lateral and vertical electrical field. The dual field plate is used to adjust the $\mathrm{N}$-drift region surface electrical field distribution.

\section{Results and Discussion}

Device simulation is performed using the TCAD software Taurus. The device structure of the $50 \mathrm{~V}$ RFLDMOS is analyzed in detail, including the dual field-plate length, Ntype lightly doped drift-region dopant concentration, and junction depth. The effect of the device DC characteristics is simulated. Based on this simulation, the device is fabricated using $0.35 \mu \mathrm{m}$ technologies; silicon data are collected and compared to confirm the accuracy of the simulation. Finally, the optimized device DC performance is obtained.

The breakdown voltage of the LDMOS device is primarily determined by the length and dopant concentration of the Ntype lightly doped drift region. When the vertical breakdown voltage is sufficiently high, the longer N-type lightly doped drift-region length results in a higher breakdown voltage and on-resistance. To reduce the on-resistance, the N-type lightly doped drift-region dopant concentration must be increased; however, in that case, the $\mathrm{N}$-type lightly doped drift region is difficult to be fully depleted, which reduces the breakdown voltage. Thus, there is a tradeoff between the breakdown voltage and on-resistance. It is obvious that obtaining better device DC performance requires considering the length and dopant concentration and junction depth of the N-type lightly doped drift-region all together. For convenience in this study, the drift-region length of the device is fixed at $5 \mu \mathrm{m}$. This paper focuses on the impact of the field-plate profile and N-type lightly doped drift-region dopant concentration on the electrical field distribution and main device DC characteristics.

3.1. The Impact of the N-Type Lightly Doped Drift-Region Concentration and Junction Depth on the Breakdown Voltage and On-Resistance. The N-type lightly doped drift-region dopant concentration and junction depth are determined by the N-type implant dosage and energy, respectively. When the length of dual field plate is fixed, the relationship of the implant dosage and energy to the breakdown voltage and onresistance can be obtained using the TCAD simulation, as illustrated in Figure 2.

As illustrated in Figure 2(a), when the field-plate structure is fixed, there is a peak value of the breakdown voltage BV versus $\mathrm{N}$-type implant dose. The implant dose corresponds to a peak $\mathrm{BV}$ which is $1.6 e 12 \mathrm{~cm}^{-2}$. As the implant dose increases, the breakdown voltage BV decreases. This finding indicates that, for a fixed field-plate structure, there is an optimized implant dose or, in other words, an optimized Ntype lightly doped drift-region dopant concentration. This result can be explained by the change in the surface electrical field distribution of the drift region. Figure 3 gives the electrical field distribution curves near the breakdown for different dopant concentrations. A few peaks appear on the surface electrical field of the drift region. Three peaks appear at the two field-plate edges and the boundary of the drift region to the drain terminal. When the dopant concentration is high, the field plate has a stronger effect on the electrical field. The peak electrical field is under the field plate, where the avalanche breakdown occurs. When the dopant concentration is low, the peak electrical field is at the boundary of the drift region to drain terminal. The area under the electrical field curve is indicative of the breakdown voltage BV. To obtain the largest breakdown voltage $\mathrm{BV}$, which corresponds to the largest area under the electrical curve, the dopant concentration should be selected properly to obtain three electrical field peaks that are more uniformly distributed. Dopant concentrations that are either too high or too low are detrimental to the final device breakdown voltage. In Figure 2(a), the breakdown voltage slightly increased when the N-type implant energy increased. When the implant energy increased, the junction depth of 


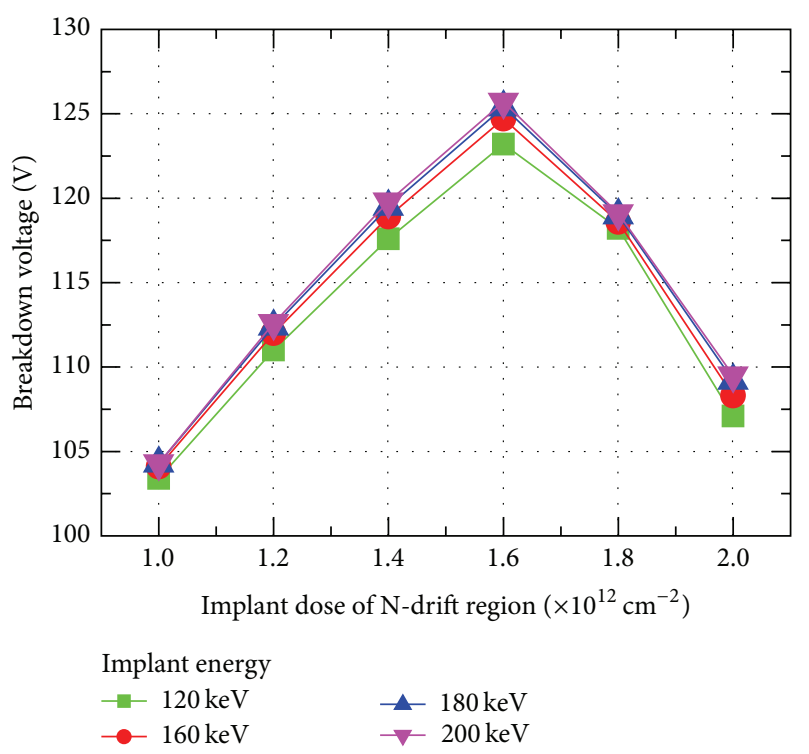

(a)

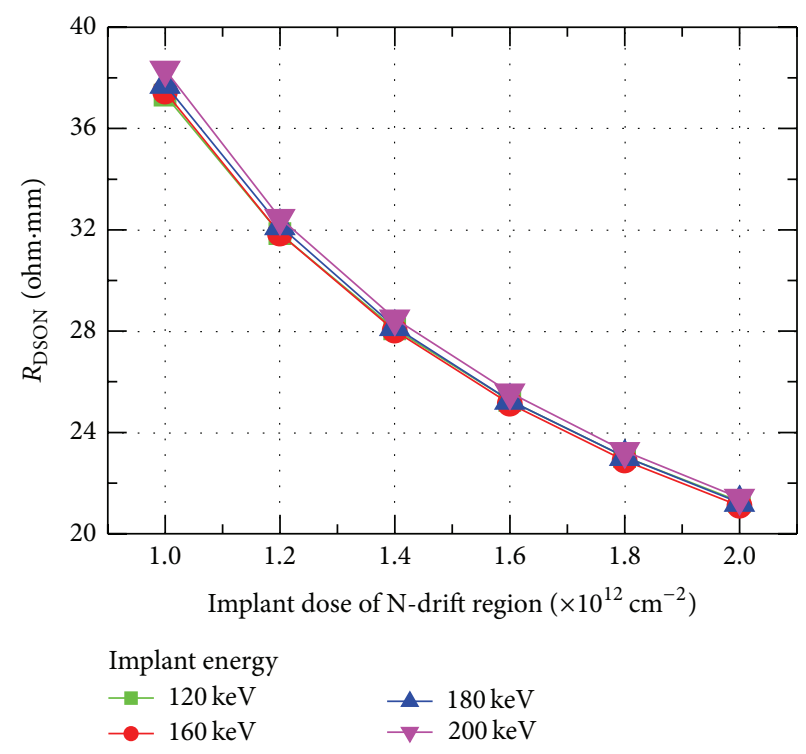

(b)

FIGURE 2: Relationship of the (a) breakdown voltage and (b) on-resistance $\left(R_{\mathrm{DSON}}\right)$ to the implant dose and energy of the N-drift region.

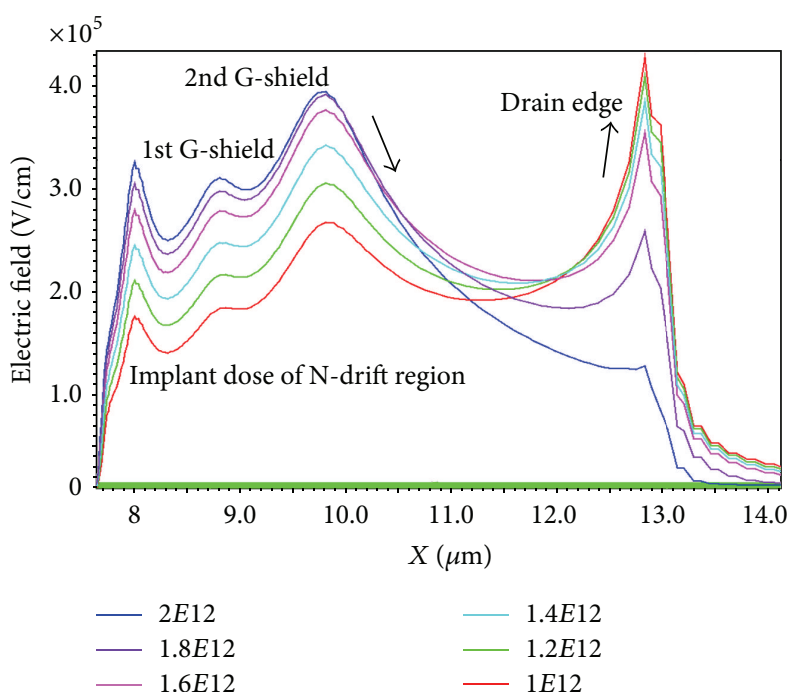

FIGURE 3: Crucial electric field distribution along the surface of $\mathrm{N}$-drift region when breakdown occurs for different given implant energies of the $\mathrm{N}$-drift region.

the N-type lightly doped drift region increased, and the surface dopant concentration decreased. This makes the electrical field distribution at the drift region surface more uniform, which helps increase the breakdown voltage.

The device on-resistance $R_{\mathrm{DSON}}$ decreases as the implant dose increases, as illustrated in Figure 2(b). They are inversely proportional because the on-resistance $R_{\mathrm{DSON}}$ is primarily determined by the dopant concentration of the $\mathrm{N}$-type lightly doped drift region. From Figure 2(b), we can also observe that the on-resistance $R_{\mathrm{DSON}}$ is less impacted by the implant energy because the carrier goes through a wide conduction path. Even if the implant energy increases, there is less change in the overall dopant concentration of the drift region.

\subsection{Impact of the Double G-Shield Structure on the Breakdown} Voltage and RDSON. The field plate of the RFLDMOS is initially used to block the gate to reduce the feedback capacitance $C_{\mathrm{GD}}$ between the drain and gate to improve the $\mathrm{RF}$ performance of the device. When the vertical distance of the field plate to the $\mathrm{N}$-type lightly doped drift region decreases, its impact on the surface electrical field distribution of the drift region becomes stronger. Thus, the field plate is also a very important factor to the device breakdown voltage. For the $50 \mathrm{~V}$ RFLDMOS device, dual field plates are adopted to obtain good RESURF effect. The profile of the dual field plate, particularly the length, determines the device breakdown voltage. The relation of the breakdown voltage to the length of the field plate is analyzed in detail for a fixed implant dose of the $\mathrm{N}$-type drift region by the TCAD simulation. The optimized field-plate dimension is obtained.

Figure 4 shows the device breakdown voltage versus the second field-plate length with the first field-plate length fixed at $0.8 \mu \mathrm{m}$. The impact of the first field plate on the device breakdown voltage will be explained later. For a fixed N-type implant dose that corresponds to a fixed drift-region dopant concentration, the breakdown voltage BV first increases, then reaches a peak value, and finally decreases as the length further increases when the second field plate increases from $1 \mu \mathrm{m}$ to $2.5 \mu \mathrm{m}$.

The optimized length of the second field plate corresponding to the peak breakdown voltage $\mathrm{BV}$ is not the same for different dopant concentrations of drift region. As shown in Figure 4, the length of the second field plate corresponding to the peak breakdown voltage is longer for a high implant dose or higher concentration of the $\mathrm{N}$-type lightly doped drift 


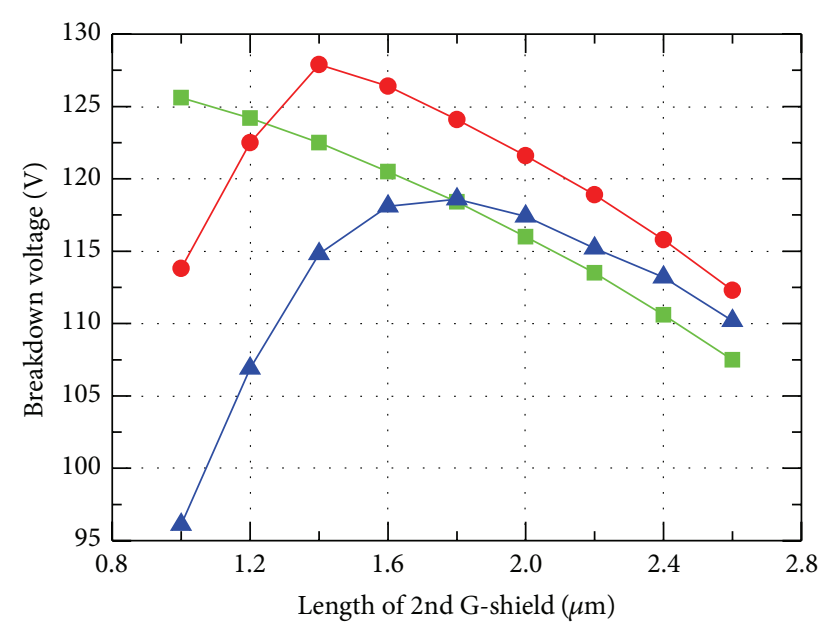

$$
\begin{aligned}
& \text { Dose } \\
& --1.4 E 12 \mathrm{~cm}^{-3} \\
& \rightarrow-1.6 E 12 \mathrm{~cm}^{-3} \\
& -1.8 E 12 \mathrm{~cm}^{-3}
\end{aligned}
$$

FIGURE 4: Relationship between the breakdown voltage and length of the 2nd G-shield with respect to different implant doses for a given implant energy of the $\mathrm{N}$-drift region.

region. If the concentration of the $\mathrm{N}$-type lightly doped drift region is low, for example, at an implant dose of $1.4 e 12 \mathrm{~cm}^{-2}$, then the length of second field plate corresponding to the peak breakdown voltage is shorter, which is close to that of the first field plate.

Figure 5(a) illustrates the lateral electrical field distribution of the N-type drift region at the voltage near breakdown when the N-type implant dose is relatively high (= $1.8 \mathrm{e} 12 \mathrm{~cm}^{-2}$ ). There are three obvious peaks at the edge of the drain and dual field plate. Because the drift region has a high dopant concentration, the peak electrical field at the second field plate is higher than the drain edge. Considering an extreme case when the second field plate is short $\left(L_{2}=\right.$ $1 \mathrm{um}$ ) and close to the first field plate, the second field plate is blocked by the first field plate because of the small distance of the first field plate to the drift region, so a very high peak electrical field exists under the field plate and very low electrical field at the drain edge. Overall, the electrical field curve is very steep, which is detrimental to obtaining a high breakdown voltage. When the length of second field plate further increases, the two peak positions of the electrical field corresponding to the two field plates gradually detach. When the second field plate is closer to the drain, it has more control over the electrical field. Its electrical field peak value is always higher than the first field plate. The strongest point of impact ionization, that is, the breakdown point, always exists under the second field plate. When the length of the second field plate gradually increases, dragged by the electrical field under the second field plate, the electrical field at the drain edge also gradually increases. When the length of the second field plate is long (i.e., $L_{2}=2.6 \mathrm{um}$ ), both the electrical fields under the second field plate and at the drain edge have high peak values. This will block the first field plate and reduce its control over the drift region. The peak value of the electrical field under the first field plate is low, and the distribution is not smooth, which corresponds to a low breakdown voltage $\mathrm{BV}$. Therefore, when the length of second field plate is $1.8 \mu \mathrm{m}$, the corresponding electrical field curve is the smoothest; the highest breakdown voltage of $118 \mathrm{~V}$ can be achieved at this dopant concentration.

Figure 5(b) shows the lateral electrical field distribution of the drift region at different second field-plate lengths when the N-type implant dose is $1.4 e 12 \mathrm{~cm}^{-2}$. The trend is similar to that observed for the higher drift-region dopant concentration. The biggest difference is that the highest peak always occurs at the drain edge. The breakdown point is also at this point because the whole drift region is fully depleted and the depletion region extends to the very high doped $n+$ region at high applied voltage. When all of the electrical lines terminate at the drain region, the drain terminal has the highest electrical field and thus breaks down first. In that case, shorter lengths of the second field plates result in higher areas under the electrical field curve and higher breakdown voltages. In such cases, there is less difference between the electrical field distribution of the dual field plates and that of single field plate.

The impact of the first field plate is not considered when discussing the impact of the profile of the dual field plates to breakdown voltage As illustrated in Figure 6, this impact of first field-plate length to breakdown voltage is minor when the second field-plate length is fixed, based on silicon data. The most obvious impact on the device characteristics is the hot carrier injection because the electrical field underneath the gate becomes stronger when the first field plate gets closer to the gate.

It is beneficial to maintain a low drift-region dopant concentration to obtain a higher breakdown voltage. However, the device on-resistance $R_{\mathrm{DSON}}$ is inversely proportional to the N-type drift-region dopant concentration. Considering both the breakdown voltage $\mathrm{BV}$ and on-resistance $R_{\mathrm{DSON}}$, it is imperative to maintain a higher $\mathrm{N}$-type drift-region dopant concentration. Thus, in order to maintain a lower on-resistance $R_{\mathrm{DSON}}$, device is optimized by utilizing the RESURF effect of dual field plate; a high breakdown voltage can be obtained by adopting an optimized field-plate dimension. It is also helpful to increase the device saturation current Idsat by keeping a higher $\mathrm{N}$-type drift-region dopant concentration.

A $50 \mathrm{~V}$ RFLDMOS dual field plate device is fabricated using $0.35 \mu \mathrm{m}$ technologies. For a fixed implant energy, the silicon data of device breakdown voltage versus the second field-plate length and $\mathrm{N}$-type drift-region dopant concentration is illustrated in Figure 7. Compared with simulation data of Figure 4, the trend observed for the measured data matches the simulation data well. There is an optimized value of the drift-region concentration and second field-plate length. The simulation data predicts the device characteristics well.

Finally, based on the simulation and experiment data, device is optimized by using the following conditions: the $\mathrm{N}$ type drift-region implant dose is $1.6 e 12 \mathrm{~cm}^{-2}$, and the second field-plate length is $1.8 \mu \mathrm{m}$, which achieves a compromise of 


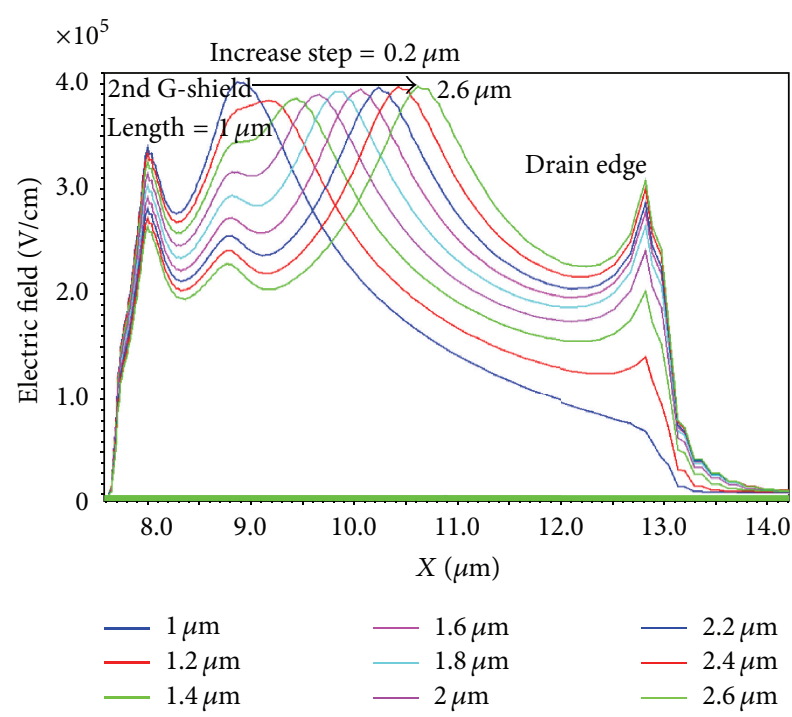

(a)

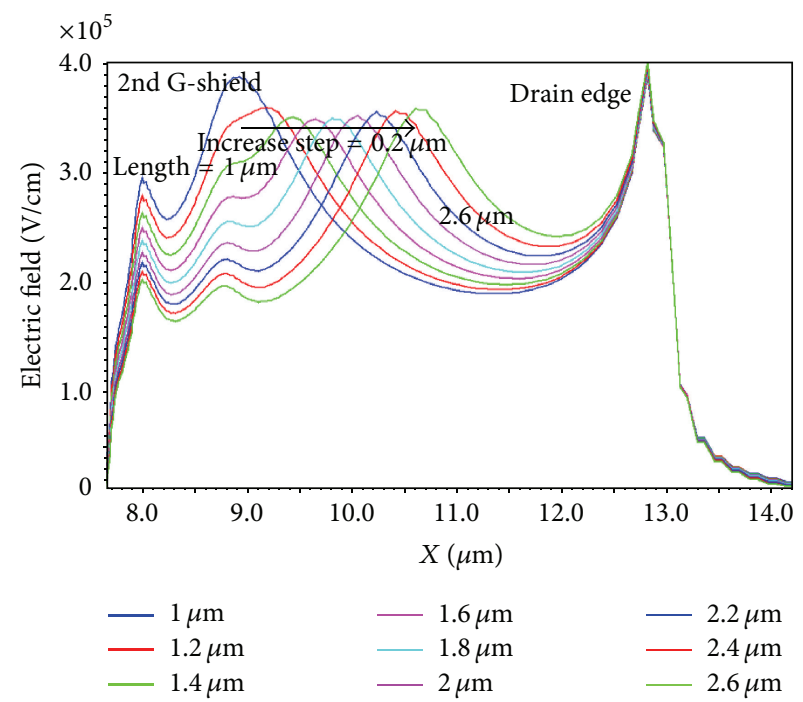

(b)

FIgURE 5: For (a) a high implant dose of $1.8 e 12 \mathrm{~cm}^{-2}$ and (b) a low implant dose of $1.4 e 12 \mathrm{~cm}^{-2}$, the crucial electric field distribution along the surface of the $\mathrm{N}$-drift region is determined by the length of the 2nd G-shield.

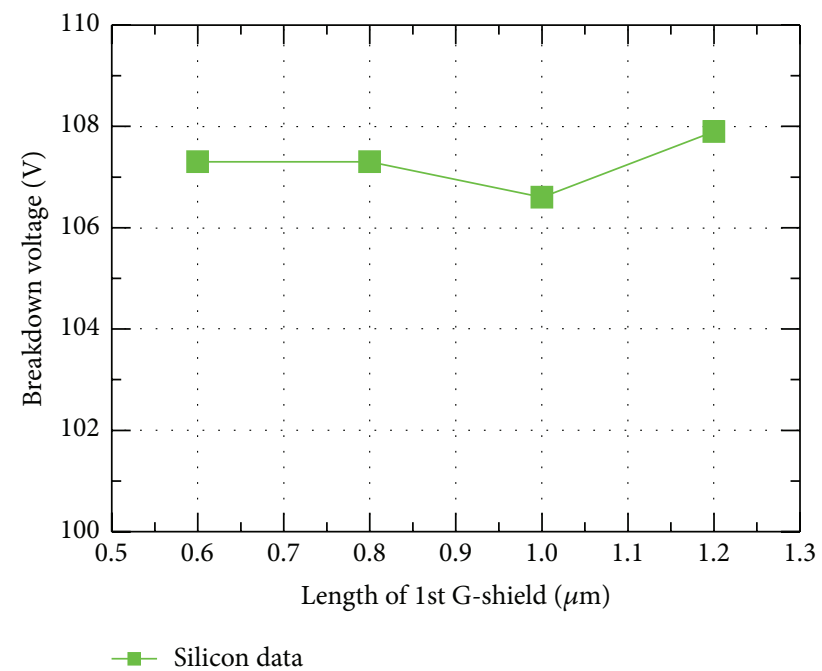

FIGURE 6: Relationship between first G-shield length and the breakdown voltage for a fixed length of 2 nd G-shield and other conditions.

the breakdown voltage and on-resistance. The competitive device performance of breakdown voltage $118 \mathrm{~V}$ and the onresistance $26 \mathrm{ohm} \cdot \mathrm{mm}$ is achieved. Figure 8 gives the device $I_{d}-V_{g}$ and $I_{d}-V_{d}$ curve. The cross-section view of the device TEM is illustrated in Figure 9.

\section{Conclusion}

The factors that affect the $50 \mathrm{~V}$ RFLDMOS DC characteristics are reviewed by performing device simulation using the TCAD software Taurus. The effects of the N-type drift-region dopant concentration, junction depth, and dimension of dual

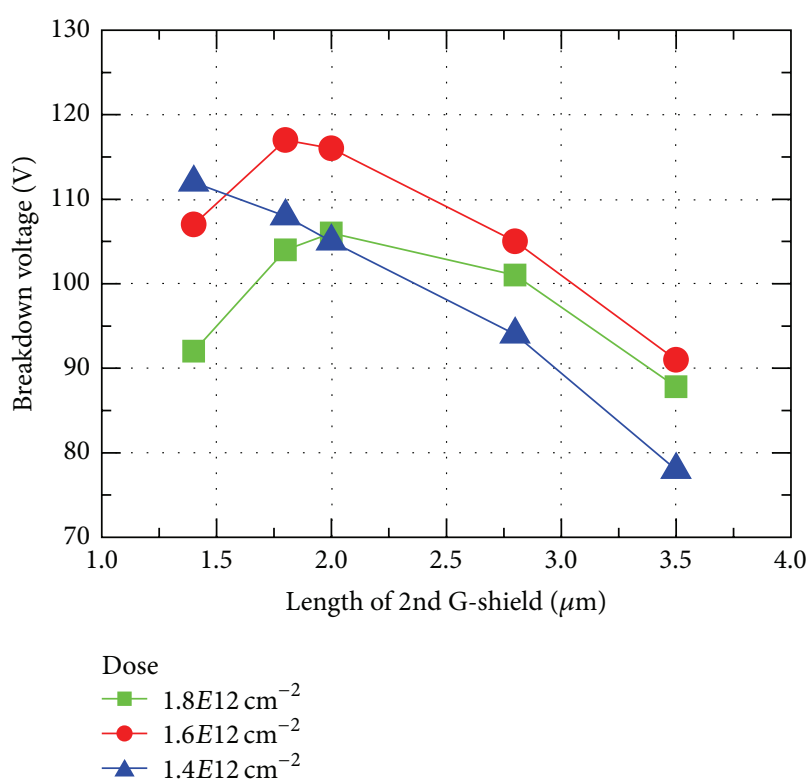

FIGURE 7: Silicon data of the effect of the 2nd G-shield length on the breakdown voltage.

field plates on the breakdown voltage BV and on-resistance $R_{\mathrm{DSON}}$ are analyzed. The real silicon data show the same trend as that of the simulation results. The junction depth of the Ntype drift region has less effect on the DC characteristics. A high $\mathrm{N}$-type drift-region dopant concentration is beneficial to obtain a lower on-resistance $R_{\mathrm{DSON}}$. However, a low Ntype drift-region dopant concentration allows for a higher breakdown voltage. There are optimum field-plate dimensions for different $\mathrm{N}$-type drift-region dopant concentrations, particularly for the second field plate. Based on simulation 


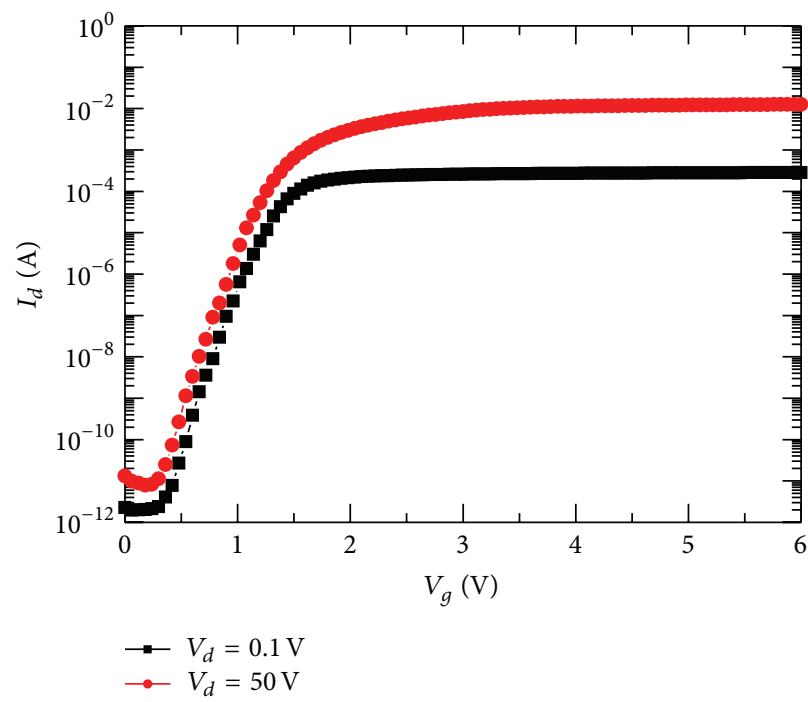

(a)

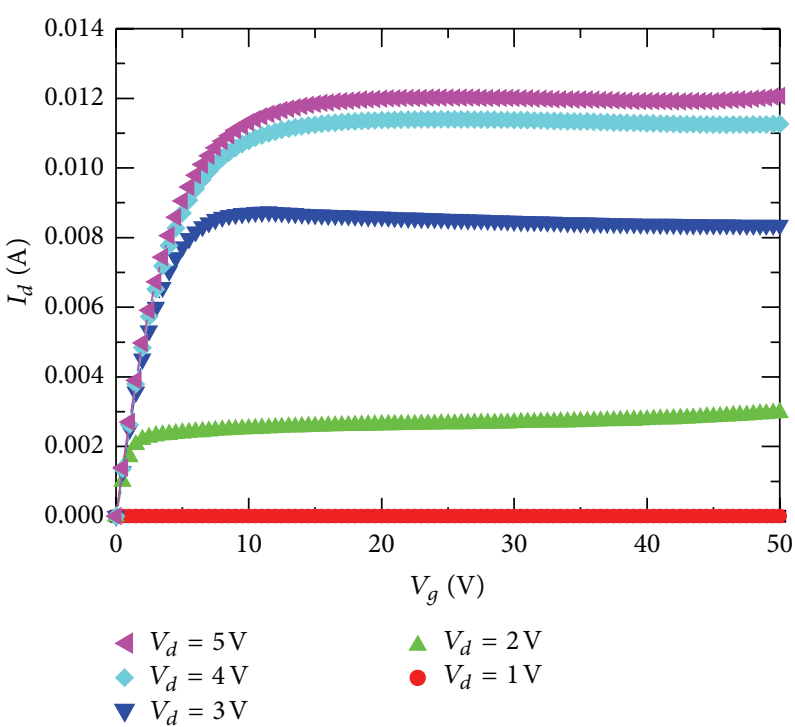

(b)

Figure 8: Optimized device characteristics (a) $I_{d}-V_{g}$ curve and (2) $I_{d}-V_{d}$ curve.

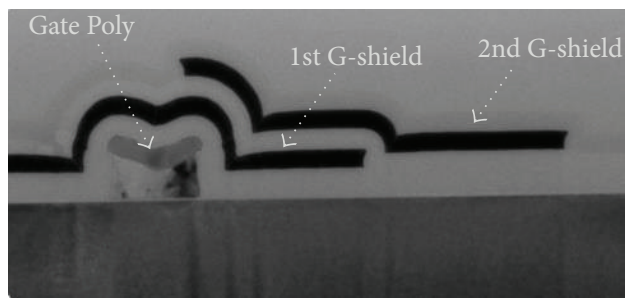

FIGURE 9: TEM picture of the optimized double G-shield RFLDMOS cross-section.

data, an experiment is performed to determine the relationship of the BV to the second field-plate length and Ntype drift-region dopant concentration. The trend from the real silicon data matches the simulation well. The device is optimized by choosing an $\mathrm{N}$-type drift-region implant dose of $1.6 e 12 \mathrm{~cm}^{-2}$ and a second field-plate length of $1.8 \mu \mathrm{m}$, which results in an excellent balance between the breakdown voltage $\mathrm{BV}$ and on-resistance $R_{\mathrm{DSON}}$. The breakdown voltage is $118 \mathrm{~V}$, and the on-resistance is $26 \mathrm{ohm} \cdot \mathrm{mm}$. Based on this result, if other factors such as the dielectrics under the field plate are also considered, the device DC characteristics can be further improved. In summary, the device structure applies the RESURF effect, and good device optimization is achieved without additional process steps. This is very useful for real applications.

\section{Conflict of Interests}

The authors declare that there is no conflict of interests regarding the publication of this paper.

\section{Acknowledgments}

The authors would like to gratefully acknowledge many colleagues of Huahong Grace Semiconductor Manufacturing Corporation for their contributions on process development, device simulation and characterization, and helpful discussions. This study is financially supported by National Program on Key Basic Research Project (973 Program) of China (Grant nos. 2011CBA00607 and 2012ZX02502).

\section{References}

[1] A. Wood, C. Dragon, and W. Burger, "High performance silicon LDMOS technology for $2 \mathrm{GHz}$ RF power amplifier applications," in Proceedings of the IEEE International Electron Devices Meeting, pp. 87-90, December 1996.

[2] H. F. F. Jos, "Novel LDMOS structure for $2 \mathrm{GHz}$ high power basestation application," in Proceedings of the European Microwave Conference, pp. 739-744, 1998.

[3] H. Brech, W. Brakensiek, D. Burdeaux et al., "Record efficiency and gain at $2.1 \mathrm{GHz}$ of high power RF transistors for cellular and 3G base stations," in Proceedings of the IEEE International Electron Devices Meeting, Technical Digest (IEDM '03), pp. 15.1.115.1.4, Washington, DC, USA, December 2003.

[4] F. Van Rijs and S. J. C. H. Theeuwen, "Efficiency improvement of LDMOS transistors for base stations: towards the theoretical limit," in Proceedings of the International Electron Devices Meeting (IEDM '06), pp. 205-208, December 2006.

[5] F. van Rijs, "Status and trends of silicon LDMOS base station PA technologies to go beyond $2.5 \mathrm{GHz}$ applications," in Proceedings of the IEEE Radio and Wireless Symposium (RWS '08), pp. 69-72, Orlando, Fla, USA, January 2008.

[6] S. J. C. H. Theeuwen and H. Mollee, "S-band radar LDMOS transistors," in Proceedings of the 4th IEEE European Microwave Integrated Circuits Conference (EUMIC '09), pp. 53-56, 2009. 
[7] D. Vye, L. Pelletier, S. Theeuwen et al., "The new power brokers: high voltage RF devices," Microwave Journal, vol. 7, pp. 22-40, 2009.

[8] K. Werner and S. Theeuwen, "RF driven plasma lighting-the next revolution in light sources are powered by solid state RF technology," Microwave Journal, vol. 53, no. 12, pp. 68-74, 2010.

[9] S. J. C. H. Theeuwen, W. J. A. M. Sneijers, J. G. E. Klappe, and J. A. M. de Boet, "High voltage RF LDMOS technology for broadcast applications," in Proceedings of the 3rd European Microwave Integrated Circuits Conference (EuMIC '08), pp. 2427, 2008.

[10] P. Piel, W. Burger, D. Burdeaux, and W. Brakensiek, "50 VRFLDMOS: an ideal RF power technology for ISM, broadcast, and radar applications," White Paper, Freescale Seminconductor, Tempe, Ariz, USA, 2011. 

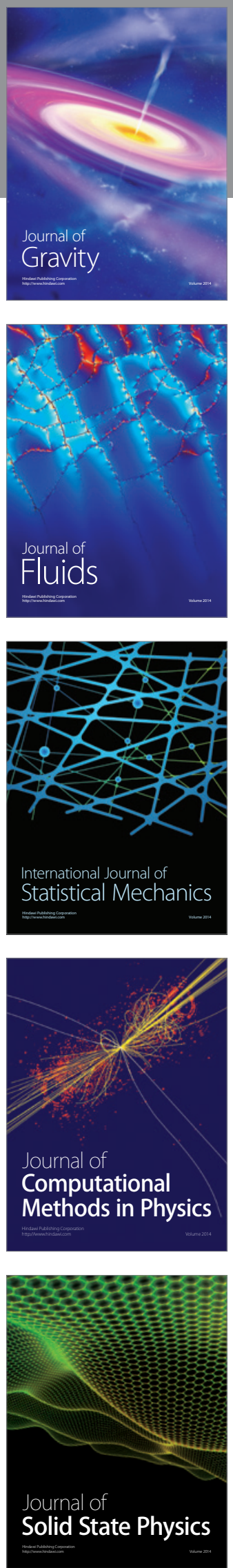

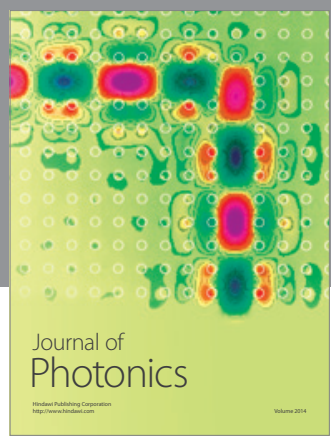

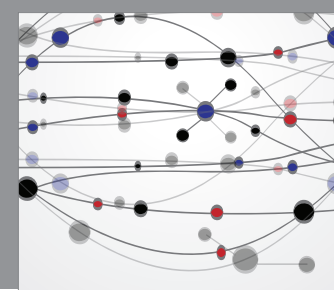

The Scientific World Journal

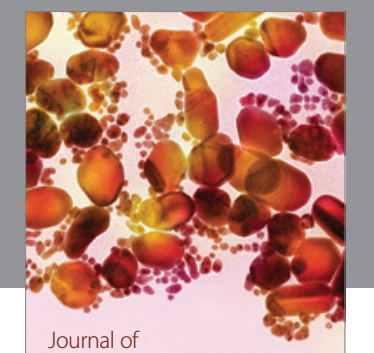

Soft Matter
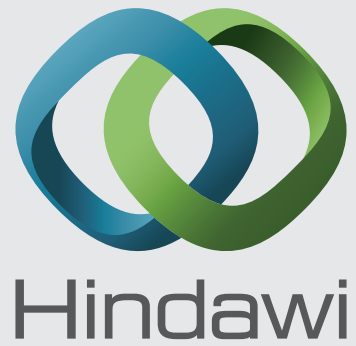

Submit your manuscripts at

http://www.hindawi.com
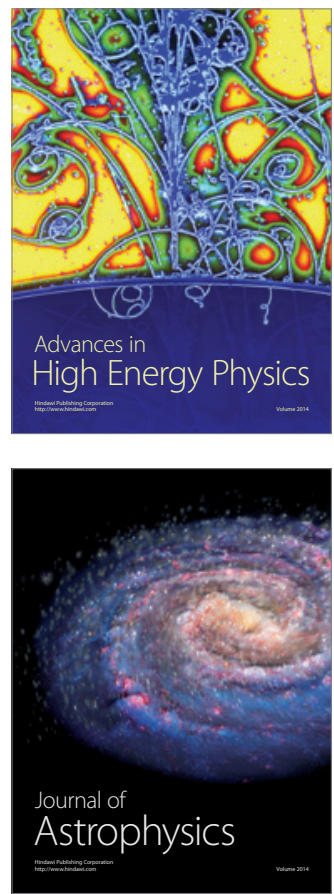
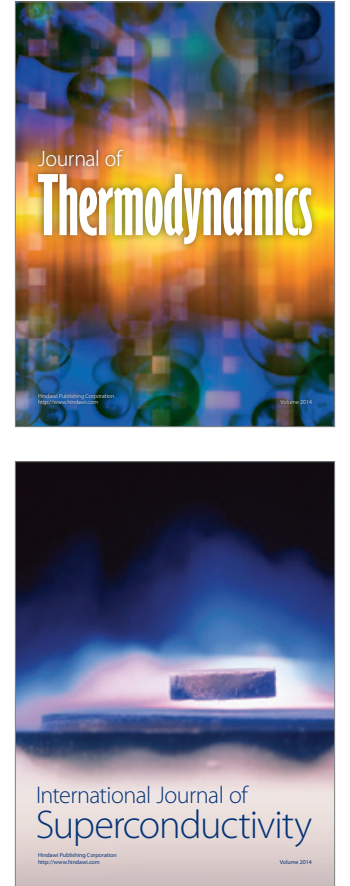
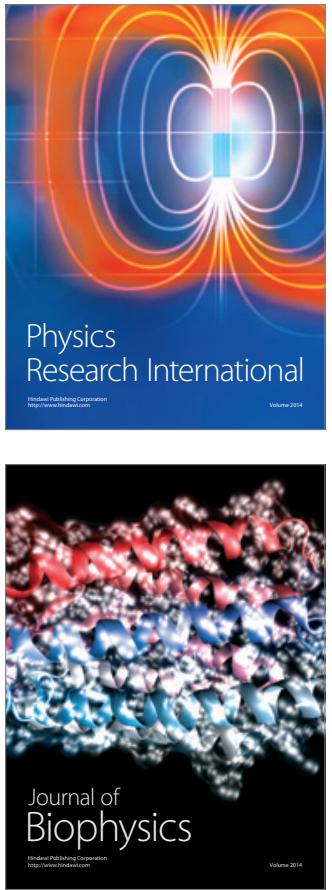
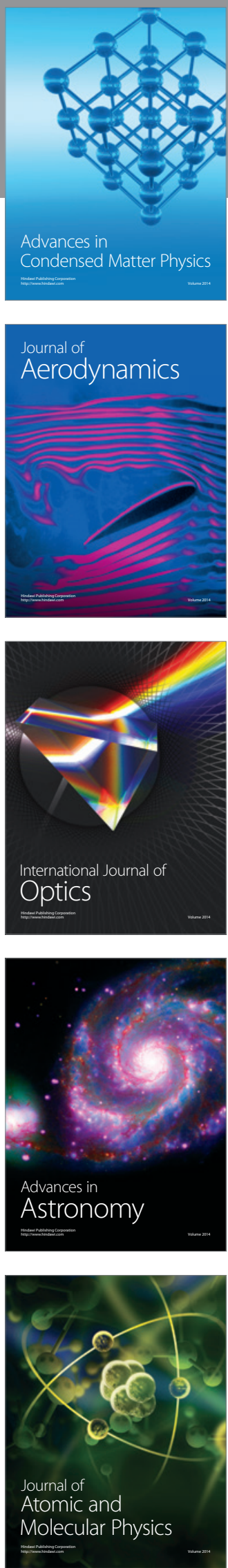